\title{
Arbor
}

\section{Habilidades morfológicas y experiencia socioeducativa}

\section{$M^{a}$ Carmen Contreras González}

Arbor CLXXIX, 705 (Septiembre 2004), 213-227 pp.

Esta investigación ha sido motivada con la intención de analizar las ejecuciones morfológicas en dos grupos de niños procedentes de dos ambientes sociales y educativos diferentes. Concretamente, un grupo de niños-as procede de un ambiente socioeducativo de clase media ( $n=33), y$ otro grupo con desventaja medioambiental, de un ambiente social de clase baja $(n=34)$, estando sus edades comprendidas entre 7 años y 6 meses, y 9 años. Los niñosas respondieron a un conjunto de subpruebas morfológicas, unas inventadas y otras reales: las primeras estaban destinadas a medir la capacidad para aplicar reglas morfológicas inventadas sin carga léxicosemántica específica, no estando mediatizadas por aprendizajes anteriores; mientras que las segundas presentaban contenido léxico semántico específico y recogían los efectos de la experiencia socioeducativa y de los aprendizajes previos. Los resultados indican que los alumnos con desventaja sociocultural no mostraban significatividad intergrupal en las primeras tareas, mientras que la situación varíaba en la segundas. Se discute la mayor dependencia socioambiental de algunas medidas morfológicas frente a otras.

\section{Introducción}

Una de las cuestiones que siempre ha llamado la atención es el rápido desarrollo del lenguaje en los niños, ya que ocurre en todas las sociedades alfabetizadas o no y en todos los idiomas independientemente del 
modo en el que éstos han sido educados, siendo uno de los factores más importantes que contribuyen al desarrollo total de la persona. Atendiendo a las explicaciones de la psicolingüística actual, hay que señalar que estos logros dependen tanto de mecanismos específicos, como de zonas cerebrales especializadas, quedando clara la pregunta qué aprenden los niños, y debatida la cuestión de cómo representamos mentalmente el lenguaje y cómo aprendemos. Así, las concepciones que asumen una visión modular del sistema de procesamiento humano preconizan el caracter autónomo del análisis gramatical respecto a otros dominios lingüísticos, mientras que las que adoptan un punto de vista interactivo justifican la importancia de la información semántica y gramatical para construir el significado de los mensajes. Por su parte, la visión lingüística generativa asume la competencia gramatical como una capacidad innata del individuo, mientras que los enfoques sociales defienden el aprendizaje social del lenguaje, y la modificabilidad de la conducta verbal en función de los mismos.

Así pues, el debate se plantea en cuanto a dos cuestiones: la primera atañe a la consideración del funcionamiento del lenguaje, bien encapsulado, o bien como una habilidad más entre otras, sujeta a procesamiento de tipo general; en otras palabras, el niño que está adquiriendo una lengua ¿interioriza un conjunto de principios cognitivos abstractos y aplica unas reglas, o aprende la lengua sin recurrir a la aplicación de reglas o paradigmas a partir de asociaciones establecidas con experiencias pasadas?; la segunda nos lleva, a su vez, hacia otro interrogante: ¿cuáles son las habilidades iniciales o predisposiciones de la especie humana para el lenguaje y cuáles son las atribuibles a los aprendizajes ambientales? Por tanto, dar una respuesta a lo planteado «implicará la comprensión no sólo de la complejidad de los sistemas lingüísticos, sino también de la dotación biológica exclusiva del niño, así como la comprensión de las características cognitivas y sociales que éstos aportan a la tarea» (Gleason y Ratner, 2001: 423).

Para revisar estas cuestiones, nos centraremos en el estudio del componente morfológico o dimensión que recoge cómo se construyen las palabras, posibilitando modificar significados mediante reglas gramaticales muy diversas. En concreto, Serra, Serrat, Sole, Bel y Aparici, (2000) indican que la gramaticalización se produce siguiendo tanto el eje lexical (por ejemplo, en los derivativos), como el sintáctico (por ejemplo, la concordancia para manifestar agrupación y dependencia;), lo que significa que no sólo es el léxico el que dirige la atención hacia los significados, sino que es el marco sintáctico y los patrones de uso gramatical, en los 


\section{Habilidades morfológicas y experiencia socioeducativa}

que se insertan las palabras (nombres, verbos, adjetivos, etc.), los que conducen hacia suposiciones sobre significados. En consecuencia, el uso de pistas sintácticas facilita una mayor adecuación de significado (Fisher, Gleitman y Gleitman, 1991) pero, a su vez, los niños usan el conocimiento inicial que poseeen de los significados léxicos para avanzar hacia el conocimiento de la estructura gramatical (Pinker, 1989).

Asimismo, las palabras disponen de una serie de entradas denominadas morfemas que facilitan acceder a las mismas y que actúan como nexos de unión entre los elementos de la oración, siendo libres lo que adquieren sentido por sí mismos y ligados en caso contrario. Entre estos últimos, se encuentran los derivativos, usados para derivar otra palabra (los prefijos al comienzo de la palabra; por ejemplo: de «hacer, «deshacer» y los sufijos al final de la palabra; por ejemplo: de «libro», «librero); y los flexivos, utilizados para indicar el número en artículos, nombres y adjetivos, así como el tiempo verbal y la concordancia entre verbo y sujeto. A diferencia de los flexivos que no alteran la estructura de la palabra (de «mesa», «mesas»), los derivativos poseen la propiedad de cambiar la categoría gramatical de una palabra en otra, siendo el caso de los cambios de verbos en sustantivos (por ejemplo: de «comer», "comida»). La organización morfémica cuenta con la ventaja del uso eficiente de recursos cognitivos, ya que no se hace necesario el almacenamiento de léxico de todas las variantes de una palabra, lo que supondría un gasto innecesario de espacio en la memoria, junto con un menor coste en el procesamiento.

Con respecto al desarrollo morfológico, Belinchón (1995: 434) asegura que la adquisición de la morfología y de la sintaxis «parece depender críticamente, en primer lugar, de la exposición a una lengua durante un periodo temprano de la vida; cuando se cumple esta condición, la construcción de los principios y categorías morfosintácticas, por parte del aprendiz de una lengua, resulta ser un proceso extremadamente robusto». En concreto, la morfología esta estrechamente ligada con la adquisición del léxico o de vocabulario que, a su vez, parece estar en dependencia de la experiencia interactiva, ya que una persona no puede recuperar el nombre de un objeto simplemente utilizando reglas, de tal forma que el léxico verbal debe alcanzar un determinado nivel o masa crítica (Marchman y Bates, 1994) para que este desarrollo tenga lugar; así se conoce que en la adquisición infantil del lenguaje, la cantidad de tiempo durante el cual la madre habla sobre temas a los que el niño ya está dedicando su atención muestra estrecha correlación con el tamaño de vocabulario inicial (Gleason y Ratner, 2001) y, que los niños que han contado con condiciones familiares y sociales que han conducido al desarro- 
llo de sus habilidades verbales (por ejemplo, la exposición al discurso narrativo), tienen ventaja en el medio escolar, ya que gran parte de las tareas escolares se centran estos aprendizajes (Garton, 1994).

$\mathrm{Al}$ contrario, un ambiente lingüístico deficiente retarda el desarrollo del lenguaje del niño (Rondal, 1990); así, la escasa complejidad de los temas de comunicación que emplean los padres de clase baja con sus hijos, determina, a su vez, usos y enunciados lingüísticos menos complejos (Tough, 1977), lo cual puede actuar limitando el proceso de alfabetización y dar lugar a un pensamiento empobrecido, siendo ambos el origen de posibles problemas escolares (Dale, 1980).

En este sentido, la caracterización de las dificultades en el componente morfológico es un paso necesario y previo de cualquier actuación dirigida a compensar las desigualdades lingüísticas de los alumnos procedentes de grupos sociales desfavorecidos (Lahey, 1990). Por lo tanto, en este trabajo se ha comparado las ejecuciones de niños-as procedentes de dos ambientes socioeducativos diferentes (normalizado y con desventaja social) con dos objetivos diferenciados:

1. Determinar la capacidad inicial de los grupos aplicando reglas gramaticales inventadas sin carga léxicosemántica específica.

2. Deteminar la influencia de los aprendizajes ambientales en tareas morfológicas reales con carga léxicosemántica procedente del contexto social.

\section{Método}

\section{Sujetos}

La muestra está compuesta por 64 niños-as de segundo y tercer curso de enseñanza primaria. Sus edades están comprendidas entre los 7 años y 6 meses y los 9 años, y su edad media es de 8 años y cuatro meses. Los participantes fueron seleccionados siguiendo los criterios de Trigo (1990) de manera que pudieran ser asignados a dos grupos con un ambiente social y educativo diferente. Así, aproximadamente la mitad de los niños-as ( $\mathrm{n}=33$; 17 niñas y 6 niños), que conforman el primer grupo (grupo A) asisten a colegios ( 4 en total) situados en barrios de clase media, proceden de familias en las que ambos padres tienen estudios (desde graduado escolar a títulos medios y superiores) y buena cualificación profesional (son obreros especializados, técnicos, funcionarios, profesores, etc.). Por su parte, el segundo grupo ( $n=34 ; 17$ niñas y 7 niños) (grupo B) 


\section{Habilidades morfológicas y experiencia socioeducativa}

asiste a colegios ( 4 en total) situados en zonas deprivadas socialmente, siendo considerados Centros de Actuación Educativa Preferente (CAEP) por la Administración Pública. Asimismo, sus padres presentan un nivel de alfabetización escaso (son analfabetos o semianalfabetos) y una baja cualificación profesional (obreros, peones, vendedor ambulante, limpiadora, o sin profesión definida). A pesar de estas diferencias los niños-as de ambos grupos no presentaban dificultades lingüísticas a juicio de sus profesores.

\section{Instrumentos}

Se aplicó un cuestionario de elaboración propia (Contreras, 2004a) compuesto por diversas medidas gramaticales: las primeras (4 en total) miden la capacidad para aplicar reglas morfológicas inventadas no aprendidas anteriormente, por lo que se considera que en éstas no interviene el efecto de los aprendizajes; mientras que las segundas (5 en total) están compuestas por medidas morfológicas reales destinadas a medir los efectos de los aprendizajes ambientales. Además, hay que señalar que se ha establecido un paralelismo entre cada medida gramatical inventada y la medida morfológica correspondiente en el ámbito real (por ejemplo, la medida de Contrarios Morfológicos Inventados tiene su control en la medida de Contrarios Morfológicos Reales). A continuación, se definen operativamente las variables:

a) Medidas morfológicas inventadas:

1. Medida de Morfología derivativa en sufijos. Consiste en la aplicación de una regla morfológica inventada en los sufijos. Se enlazaría con dos pruebas reales: Medida de Asociaciones derivadas en nombres y Medida de Adverbializaciones. Pretende medir la capacidad para aplicar reglas gramaticales en sufijos. Consta de 10 items. Un ejemplo de ítem es el siguiente: «Transforma esta palabra terminándola en "ación». Por ejemplo, empate..empatación...etc.

2. Medida de Morfología derivativa en Contrarios. Se basa en la capacidad para transformar los prefijos de modo que signifiquen lo contrario. Se corresponde con la prueba de Contrarios morfológicos con palabras reales. Esta destinada a medir las destrezas del sujeto para aplicar reglas gramaticales con los prefijos. Consta de 10 items, uno de los cuales es el siguiente: "Cambia las palabras que empiecen por "des» por "dis» para que signifiquen lo contrario. Por ejemplo, "desempate, disempate».

3. Medida de Morfología flexiva en Marcadores de género y número. El sujeto debe aplicar flexiones de género y número inventadas en los sufijos. Mide la capacidad para 


\section{$M^{a}$ Carmen Contreras González}

establecer concordancia de género y número dentro del sintagma nominal. También tendría correspondencia con una medida real de morfología; Consta de 10 items, uno de los cuales es el siguiente: «Arregla las expresiones que te diré. Por ejemplo, «este niñas»...".

4. Medida de Morfología flexiva en Regularización de verbos. Consiste en aplicar reglas morfológicas flexivas a verbos. Tendría su prueba paralela en la de Flexiones verbales reales. Con ella se pretende medir la destreza para realizar flexiones en verbos. Consta de 10 items, uno de los cuales es el siguiente: "Vamos a hablar como los pequeños. Por ejemplo, «Si yo digo, dormir», tu dirás... yo dormo,...».

\section{b) Medidas morfológicas reales}

1. A. Medida de Asociaciones derivativas en nombres. Considera el total de derivaciones que realiza el sujeto a partir de una palabra estímulo, esto es, la aplicación se realiza en los sufijos de las palabras reales. Por lo tanto, mide los aprendizajes realizados en nombres derivados. Consta de 5 palabras estímulos o items, uno de los cuales es el siguiente: «Díme palabras que empiecen por «zapato». Por ejemplo, «zapatería»...». La puntuación total vendrá dada por el número total de nombres derivados sobre las palabras estímulo, otorgándose un punto a cada uno de ellos.

1.B. Medida derivativa de Adverbializaciones. El sujeto debe añadir a una palabra estímulo un sufijo «mente», de manera que el adjetivo se transforme en adverbio. Mide los aprendizajes realizados por los participantes en cuanto a las adverbializaciones, de manera que la categoría gramatical de la palabra cambia de adjetivo a adverbio. Consta de 10 items, uno de los cuales es el siguiente: «Debes expresar otra palabra diferente terminada en «mente». Por ejemplo, si yo te digo «posible», tú me dices «posiblemente»..etc.

2. Medida derivativa de Contrarios morfológicos. El sujeto tiene que expresar ante una palabra estímulo, otra que signifique lo contrario utilizando un prefijo. Mide los aprendizajes de los antónimos realizados por los participantes. Así, las palabras ofrecidas por el examinador deben ser transformadas en otras con significado contrario. Consta de 10 items, uno de los cuales es el siguiente: "Tienes que expresarme la palabra contraria a la que te diga poniéndole algo delante. Por ejemplo, si yo te digo "posible», tú me dices "iimposible»...".

3. Medida flexiva nominal de Concordancia de género y número. Consiste en la concordancia de género y número que el sujeto ha de establecer entre determinante y núcleo en el sintagma nominal: Mide los aprendizajes realizados en estos aspectos. Consta de 6 items, uno de los cuales es el siguiente: «Arregla las expresiones que te diré. Por ejemplo, «este niñas», será «estas niñas.».

5. Medida de Flexiones verbales. Se caracteriza porque el sujeto debe adaptar el verbo en infinitivo al número y persona del sujeto, a la vez que debe establecer adecuación temporal con el adverbio o referencia temporal que aparece en la frase. Mide los aprendizajes referidos a las flexiones verbales. Consta de 6 items, uno de los cuales es el siguiente: «Arregla estas frases. Por ejemplo «nosotros comprender ayer"...»Nosotros comprendimos ayer». 


\section{Procedimiento de evaluación y de corrección de las medidas}

En este trabajo hemos empleado una grabadora, un cronómetro, las medidas ya comentadas y su material gráfico correspondiente. Todos los niños-as fueron evaluados individualmente por la primera autora en una habitación sin ruidos para evitar distracciones. Antes de aplicar las medidas se preguntaba a los niños-as sobre los ejemplos incluidos en las instrucciones de cada una de ellas con el fin de asegurarnos de que los entendían. A cada ítem se le ha otorgado un tiempo máximo de respuesta de 5 segundos. Como paso previo a la evaluación, se ha procurado interaccionar con los niños-as a fin conseguir un ambiente relajado, a la vez que se ha realizado una ficha de control en la que se recogen datos personales, familiares y escolares habituales en este tipo de investigación (p.e., nombre y apellidos de los niños-as, fecha de nacimiento, número de hermanos, lugar que ocupa en la familia, profesión y estudios realizados por ambos padres, centro escolar, etc.). En lo que respecta a la corrección hay que señalar que cada ítem correcto se puntuaba con un punto, mientras que los incorrectos y la omisión de respuestas con 0 puntos, siendo la puntuación final la suma de todos los puntos de la subprueba.

\section{Resultados}

Para analizar los resultados obtenidos en este estudio, hemos realizado análisis multivariados multivariados (MANOVA) de las medidas o variables pertenecientes a los distintos dominios lingüísticos, con el grupo de procedencia de los niños-as (grupo A: ambiente socioeducativo medio; o grupo B: ambiente socioeducativo deprivado o bajo) como factor de agrupación. Si los resultados eran estadísticamente significativos, se procedía a analizar los resultados de los ANOVAS para cada variable.

En la tabla 1a y 1b pueden observarse las medias y desviaciones típicas de cada una de las variables de sintaxis medidas (Ver gráficos 1 y 2). 
TABLA $1 \mathrm{a}$

Medias y desviaciones típicas en las medidas de morfología inventada en ambos grupos de niños-as (grupo A: ambiente socio-educativo medio; grupo B: ambiente socio-educativo deprivado o bajo)

\begin{tabular}{|l|c|c|c|c|}
\hline \multirow{2}{*}{\multicolumn{1}{|c|}{ Variables }} & \multicolumn{2}{c|}{ GRUPO A } & \multicolumn{2}{c|}{ GRUPO B } \\
\cline { 2 - 5 } & X & DT & X & DT \\
\hline Medida de Morfología derivativa en sufijos & 7,24 & 2,45 & 7,71 & 1,93 \\
\hline Medida de Morfología derivativa en prefijos & 8,91 & 1,72 & 9,03 & 1,36 \\
\hline Medida de Morfología flexiva en Marcadores de género y número & 8,27 & 2,27 & 7,65 & 2,44 \\
\hline Medida de Morfología flexiva en Regularización de verbos & 6,24 & 2,83 & 6,38 & 2,52 \\
\hline
\end{tabular}

TABLA $1 \mathrm{~b}$

Medias y desviaciones típicas en las medidas morfológicas reales en ambos grupos de niños-as (grupo A: ambiente socio-educativo medio; grupo B: ambiente socio-educativo deprivado o bajo)

\begin{tabular}{|l|c|c|c|c|}
\hline \multirow{2}{*}{\multicolumn{1}{c|}{ Variables }} & \multicolumn{2}{c|}{ GRUPO A } & \multicolumn{2}{c|}{ GRUPO B } \\
\cline { 2 - 5 } & $\mathbf{X}$ & $\mathbf{D T}$ & $\mathbf{X}$ & DT \\
\hline Medida de Asociaciones derivativas en concordancia nominal & 12,06 & 4,27 & 9,56 & 3,85 \\
\hline Medida derivativa de Adverbializaciones & 9,00 & 1,52 & 7,76 & 1,92 \\
\hline Medida derivativa de Contrarios morfológicos & 7,94 & 1,46 & 5,91 & 2,08 \\
\hline Medida flexiva de Concordancia de género y número & 5,91 & 0,29 & 5,75 & 0,73 \\
\hline Medida de Flexiones verbales & 5,06 & 0,97 & 3,68 & 0,98 \\
\hline
\end{tabular}

El MANOVA de estas variables, con el grupo de procedencia de los niños-as como factor de agrupación, no arroja significatividad en las diferencias cuando se trata de Pruebas Morfológicas Inventadas en sus 4 elementos. Por lo tanto, la capacidad para aplicar estas reglas gramaticales sin carga semántica específica no parece acusar los efectos de los aprendizajes.

No obstante los resultados han sido muy diferentes en las Pruebas morfológicas Reales, esto es, cuando han intervenido los efectos de los aprendizajes. El MANOVA de estas variables, con el grupo de proceden- 
Habilidades morfológicas y experiencia socioeducativa

cia de los niños-as como factor de agrupación, arroja diferencias estadísticamente significativas entre los dos grupos: $\mathrm{F}(15,51)=4.458 ; \mathrm{p}=0.001$.

Como puede verse en las tablas de medias y de diferencias significativas, se confirma que éstas son favorables para el grupo de alumnos-as procedentes de clase media cuando intervienen los efectos de los aprendizajes en las tareas, con la salvedad de una medida en la que no se aprecian los efectos del ambiente (Medida de concordancia de género y número), lo que puede ser debido posiblemente a la automaticidad que implica la tarea.

Además de este efecto general, la tabla 2 recoge los efectos simples (ANOVAS) encontrados en las variables utilizadas entre ambos grupos.

TABLA 2

Resultados de los ANOVAS en cada variable morfológica y significatividad de las diferencias entre ambos grupos de niños-as

\begin{tabular}{|l|c|c|}
\hline \multicolumn{1}{|c|}{ Variables } & $\mathbf{F}(\mathbf{1 5 , 5 1 )}$ & $\mathbf{p}$ \\
\hline Medida de Asociaciones derivativas en nombres & 6,342 & 0,014 \\
\hline Medida derivativa de Contrarios morfológicos & 21,248 & 0.000 \\
\hline Medida derivativa de Adverbializaciones & 8,469 & 0,005 \\
\hline Medida de Flexiones verbales & 34,008 & 0,000 \\
\hline
\end{tabular}

Por otra parte, se aprecian también niveles diferentes de significatividad dentro de las variables con diferencias. Así, las dificultades se constatan en la medida de Asociaciones derivadas (nombres), se hacen más acusadas cuando estas asociaciones derivativas cambian de categoría, esto es, el adjetivo se transforma en adverbios (Medida de adverbializaciones), y adquieren relevancia en las medidas de Contrarios Morfológicos y de Morfología verbal, lo que puede orientarnos de que las diferencias aumentan según el carácter abstracto y formal de las medidas.

\section{Discusión}

El objetivo fundamental de este trabajo era arrojar luz sobre las diferencias morfológicas de dos grupos de niños-as que encuentran en etapas avanzadas del desarrollo del lenguaje, pero que proceden de ambientes 


\section{$M^{a}$ Carmen Contreras González}

socioeducativos muy diferentes (medios y desfavorecidos respectivamente), para determinar las capacidades para el aprendizaje reglado, así como la influencia de los aprendizajes ambientales, con vistas a la aplicación de programas educativos específicos compensadores de posibles dificultades lingüísticas.

De acuerdo con los resultados obtenidos, se puede decir que las únicas variables en las que no existen diferencias significativas intergrupales han sido las relacionadas con tareas de aplicación de reglas morfoló gicas no aprendidas anteriormente, y que no suponen carga léxicosemántica específica, esto es, se confirma que los participantes han aplicado paradigmas sin diferencias significativas, lo que supone unas implicaciones teóricas y practicas:

La capacidad en ambos grupos para el aprendizaje reglado o paradigmático apoya los planteamientos de que las personas aprendemos el lenguaje aplicando reglas gramaticales (Pinker, 1984), siendo la organización morfémica un recurso cognitivo de carácter económico que ahorra el almacenamiento de todas las variantes de una palabra. (Taft, 1981; Taft y Foster, 1975, 1976; citados por Reeves, Hirsh-Pasek y Golinkoff, 2001). En concreto, las palabras están formadas por morfemas constituyentes, esto es, cuando escuchamos el habla de una persona, despojamos una palabra de sus afijos y activamos la raíz, junto con los morfemas ligados relevantes, lo que precisa menor número de unidades léxicas en el almacenamiento de léxico.

La presencia de capacidades iniciales para aplicar reglas gramaticales indica que, al igual que los niños de clase media, los niños del grupo desfavorecido cuentan con un adecuado acceso a la representación gramatical, lo que no supondrá un obstáculo para el aprendizaje de léxico o de términos asociados (Belinchón, Igoa y Riviere, 1992); por lo tanto, ofreciendo una estimulación adecuada, estos niños pueden mejorar en breve tiempo su competencia morfológica.

Por el contrario, los resultados han sido diferentes y muestran significatividad en el grupo de clase media en tareas morfológicas aprendidas, de lo que se deducen también consecuencias para la investigación:

Se confirma que una vez establecido control intergrupal sobre la capacidad reglada, los aprendizajes morfológicos se hallan en estrecha dependencia de la historia educativa previa y del entorno ambiental; en consecuencia, la representación gramatical de la palabra, junto con la información que ofrece la situación de interacción ayudan para que haya un adecuado acceso a la misma y, cuanto más propicios sean los estímulos ambientales, los aprendizajes serán mejores. Asimismo, dado que la 


\section{Habilidades morfológicas y experiencia socioeducativa}

morfología integra aspectos sintácticos y semánticos se reanuda, de nue vo, la polémica sobre la ubicación de la misma, una cuestión muy debatida hasta el momento.

Las interacciones sociales recibidas por los niños desfavorecidos se manifiestan en unas producciones morfológicas más pobres por desconocimiento de léxico, esto es, dado que la estimulación ambiental es menor, sus producciones han sido más escasas. Así, se presupone que los padres de clase media utilizan temas de conversación que inducen a usos más complejos en el lenguaje (Tough, 1977), lo que hace que las formas gramaticales empleadas y el léxico también lo sean; si tenemos en cuenta que el nivel de alfabetización de los padres, el trabajo de los mismos, la vivienda y el barrio, etc., varía apreciablemente entre ambos grupos de participantes, se comprende que los niños de clase social baja utilizan su lengua en un contexto que no precisa de un elevado grado de complejidad de ésta, pero esto no significa que esta capacidad no se encuentre a su alcance.

Por otra parte, hay que señalar que no se observa el mismo grado de significatividad desfavorable del grupo de clase baja con respecto al de clase media. Así, confirmamos, por una parte, que el cambio de la propiedad de una palabra (Medida de adverbializaciones) acentúa la significación con respecto a su permanencia en la misma categoría (Medida de Morfología derivativa en nombres); por otra, que la presencia de palabras de carácter abstracto (Contrarios Morfológicos), de carácter procesual o de eventos que se transforman en el tiempo (Flexiones verbales) implican una complejidad lingüística acentuada que es muy posible que no esté disponible en el contexto socioeducativo de estos niños, lo que confirma que la adquisición de formas verbales, por parte del aprendiz de una lengua, se ve obstaculizada cuando hay carencia de oportunidades para llevarla a cabo.

En general, y de cara a la vertiente aplicada, se deduce que es preciso valorar no sólo la capacidad de los sujetos para el aprendizaje reglado, sino también los aprendizajes resultantes del contexto ambiental, para determinar con mayor precisión si estamos ante un problema relativo a la falta de competencia gramatical, como es el caso de las patologías específicas del desarrollo del lenguaje (Gopnik y Crago, 1991; Ullman y Gopnik, 1999), o ante un problema de desconocimiento de palabras por falta oportunidades socioeducativas (Tough, 1977; Lahey, 1990).

En suma, la habilidad para aplicar reglas gramaticales, demuestra que ambos grupos de participantes han internalizado el conocimiento de reglas morfológicas de su lengua, es decir, no lo han hecho a base de ex- 
periencias memorísticas (Berko, 1958). Estos resultados apoyan los planteamientos de que los niños realizan generalizaciones a partir de una capacidad mental que les permite extraer las reglas de la gramática a partir de los estímulos que reciben, y el hecho de que lo hagan sin necesitar apenas ayuda indica que están empleando otra habilidad diferente a la inteligencia general (Jakendoff, 1997; citado por Serra Serrat, Solé, Bel y Aparici, 2000). Concretamente, Lahey (1990) citando la teoría de la inteligencias múltiples de Gadner (1985), en la que el autor sugiere varios tipos de inteligencia con distintos grados de autonomía, sostiene que las medidas en tests de inteligencia no están motivadas en términos de desarrollo cognoscitivo del lenguaje, por lo que no representan su desarrollo real (Fletcher e Ingham (1996).

No obstante, a la luz de los resultados también confirmamos, que, además de presencia de capacidades para el aprendizaje reglado, se constatan los efectos de los aprendizajes. La interacción social se aprovecharía de estas habilidades o recursos de la especie para el aprendizaje del sistema comunicativo de una lengua. Por tanto, esto no nos llevaría a conclusiones de modularidad estricta, sino a considerar que los medios para procesar el lenguaje deben estar relacionados con los estímulos provenientes del medio social. En otras palabras, las predisposiciones específicas para el dominio del lenguaje canalizan el desarrollo temprano del lenguaje niño al interactuar de manera compleja con los estímulos ambientales, de manera que dicha interacción influye a su vez en el desarrollo cognitivo para poder comprender y expresar nuevos significados y estructuras, de manera que se produce una reorganización del propio sistema (Karmiloff-Smith, 1992).

En resumen, estos resultados permiten constatar que las destrezas gramaticales analizadas no se manifiestan de la misma forma, ya que las tareas morfológicas sin carga léxicosemántica específica muestran escasa dependencia de la historia educativa previa y de la influencia ambiental (Fodor, 1983; Pinker, 1995); mientras que las tareas morfológicas aprendidas, evidencian estrecha relación con respecto a la experiencia socioeducativa y al entorno ambiental por la inclusión de rasgos semánticos en el léxico, lo que refleja que el aprendizaje de las palabras no tiene sentido fuera del contexto interactivo (Garton, 1994).

Por tanto, todas estas observaciones revisten gran importancia en cuanto a la respuesta socioeducativa de la enseñanza compensatoria, esto es, aceptar la posición de que se transmiten unos conocimientos cuando falla herramientas básicas de aprendizaje, lejos de ayudar empeora las diferencias sociales. Si consideramos que en las tareas escolares 


\section{Habilidades morfológicas y experiencia socioeducativa}

de las etapas educativas más avanzadas se intensifican las demandas cognitivas con respecto a la conceptos abstractos y se requiere una rápida recuperación de palabras, deducimos la necesidad de insistir en estos aprendizajes básicos, para evitar que la pobreza de recursos léxicos y morfológicos de estos niños-as impacten los aprendizajes más académicos propios de la enseñanza secundaria (Vellutino, 1979), especialmente, cuando su origen no estriba en falta de capacidades gramaticales.

En conclusión, el acceso a los aprendizajes morfológicos implica una representación de recursos lingüísticos, cognitivos y sociales, con la consiguiente economía de recursos y la enorme ampliación de posibilidades. Por consiguiente, hay que señalar que resulta necesario seguir profundizando en el estudio de la morfología, así como en las habilidades e influencias que la hacen posible, para comprender y mejorar las dificultades sociales y académicas que los alumnos puedan experimentar, ya que gracias a la palabra ampliarán su experiencia de manera extraordinaria.

GRÁFICO 1

Medias en medidas morfológicas inventadas

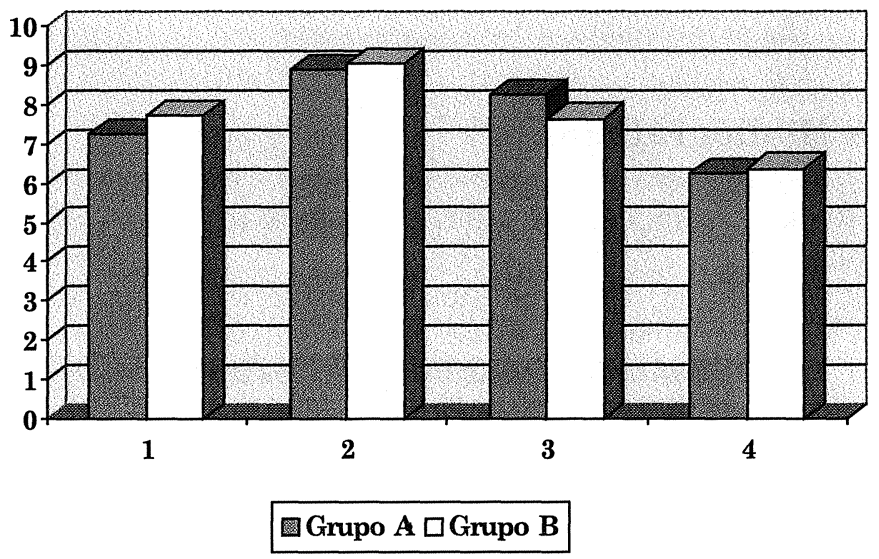

Leyenda:

1. Medida de morfología derivativa en sufijos.

2. Medida de morfología derivativa en prefijos.

3. Medida morfológica flexiva de género y número.

4. Medida morfológica flexiva en regularización de verbos 
GRÁFICO 2

Medias en medidas morfológicas reales

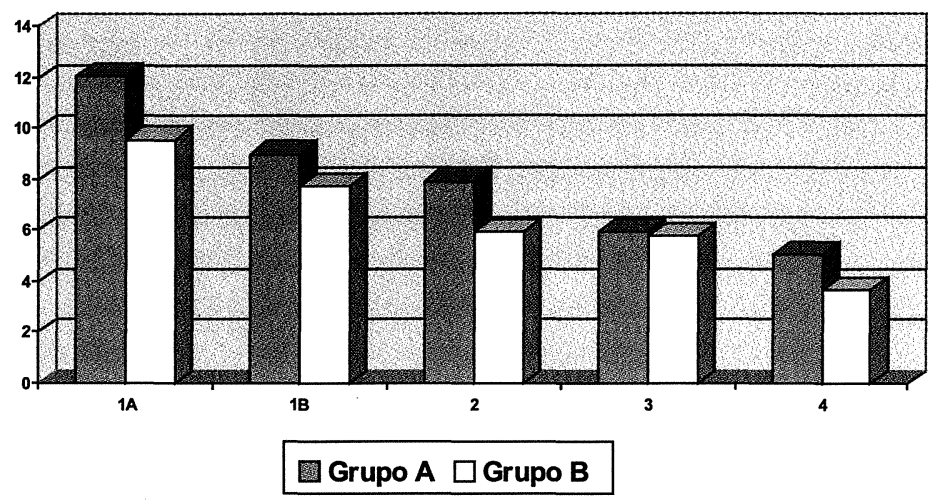

Leyenda:

1. A. Medida de asociaciones derivativas en nombres.

2. B. Medida derivativa de adverbializaciones.

3. Medida derivativa de contrarios morfológicos.

4. Medida nominal flexiva de concordancia en género y número.

5. Medida de flexiones verbales.

\section{Referencias Bibliográficas}

Belinchon, M. Riviere, A. E IGOA, J.M. (1992): Psicología del lenguaje. Investigación y teoría. Madrid, Trotta.

BELINCHON, M.(1995): «Autonomía de la sintaxis y patologías del lenguaje: datos y controversias». En M. M. Fernández y A. Anula (eds.), Sintaxis y Cognición. Madrid, síntesis.

CONTRERAS, M. C. (2004): Dificultades lingüísticas en los trastornos del lenguaje frente a la desventaja medioambiental. Tesis Doctoral. Granada: Editorial Universidad de Granada.

Fernández, M. \& ANUla A.(eds.): Sintaxis y Cognición.Madrid: Síntesis.

Berko, J. (1958): The chil'learning of English morphology. Word, 14, 150-177.

Berko Gleason, J. \& Bernstein Ratner, N. (2001): La adquisición del lenguaje. En J. Berko y N. Bernstein, Psicolingüistica (369-426). Madrid: McGraw Hill.

Bernstein Ratner, N. Gleason, J. \& NARAsimhan, B. (2001): Una introducción a la psicolingüística: ¿Qué saben los hablantes? . En J. Berko y N. Bernstein, Psicolingüísti$c a$ (pp 1-51). Madrid: McGraw Hill.

Dale, P. S. (1980): Desarrollo del lenguaje. Mexico:Trillas (ed. española).

Fisher, C. Gleitman, L. \& Gleitman, H. (1991): On the semantic content of subcategorization frames. Cognitive Psychology, 23, 331-392.

Fletcher, P. \& INGHAM, R. (1996): «Grammatical impairment». En P. Fletcher y B. MacWinney (eds.) The Handbook of child language. Oxford, Blackwll Publishers, Ltd. (pp: 603-622) 


\section{Habilidades morfológicas y experiencia socioeducativa}

FoDOR, J. A. (1983): La modularidad de la mente. Madrid: Morata.

GARTON, A. F. (1994): Interacción social y desarrollo del lenguaje y la cognición. Barcelona: Paidós.

GoPNIK, M. \& CRAGO, M. B. (1991): Familial aggregation of a developmenttal language disorder. Cognition, 39, 1-50.

KARMILOF-SMITH, A. (1992): Más allá de la modularidad. Madrid: Alianza.

LAHEY, M. (1990): Who shall be called language disordered? Some reflections and one perspective. Journal of Speech and Hearing Disorders, 55, 612-620.

MARCHMAN, V. \& BATES, E. (1994): Continuity in lexical and morphological development: a test of the critical mass hypothesis. Journal of child language, 21, 339-366.

OKSAAR, E. (1983): Language adquisition in the early years: An introduction to paedolinguistics. Londres: Brastford Academic and Educational Ltd.

PINKER, S. (1989): Learnabality and cognition: the adquistion of argument structure, Cambridge, MA: MIT Press.

PinkeR, S. (1995): El instinto del lenguaje. Madrid: Alianza.

ReEves, L., HiRsh-PASEK, K. \& GolinkofF, R. (2001): «Palabras y significado: de los elementos simples a la organización compleja». En J. Berko y N. Bernstein, Psicolingüística (pp: 169-238). Madrid: McGraw Hill.

RoNDAL, J. A. (1990): La interacción adulto-niño y la construcción del lenguaje. México: Trillas.

Serra, M. Serrat, E. Solé, R. Bel, A. \& Aparici, M. (2000): La adquisición del lenguaje. Ariel Psicología: Barcelona.

Snow, C. E., Arlmann-Rupp, A., Hassin, J., Jobse, Y., Joosten J. \& Voster, J. (1976): Mother's speech in three social classes. Journal of Psycholingüistic Research, 5, 1- 20.

Tough, J. (1977): The development of meaning. Nueva York: Halsted Press.

TRIGo, J. M. (1990): El habla de los niños en Sevilla. Sevilla: Alfar.

ULlMAN, M. T. \& GoPNIK, M. (1999): Inflectional morphology in a family with inherited specific language impairment. Applied Psycholinguistics, 20, 51-117.

Vellutino, (1979): Dyslexia. Theory and Research. Cambridge, Ma: MIT Press. 